\title{
Structural results for quaternionic Gabor frames
}

\author{
Paula Cerejeiras, Stefan Hartmann and Heikki Orelma
}

\begin{abstract}
We study quaternionic Gabor frames based on the two-sided quaternionic windowed Fourier transform. Since classical Hilbert space based methods do not work in this case we introduce appropriated versions of translation and modulation operators. We prove Janssen's and Walnut's representations, as well as modified versions of the Wexler-Raz biorthogonality and Ron-Shen duality based on the concept of correlation function. We end up with a characterization of tight quaternionic Gabor frames.
\end{abstract}

Mathematics Subject Classification (2010). Primary 42C15; Secondary $30 \mathrm{G} 35$.

Keywords. Quaternionic Gabor frames, two-sided QWFT, quaternionic frame operator, Janssens representation, Wexler-Raz biorthogonality, frame bounds.

\section{Introduction}

The two-sided quaternionic windowed Fourier transform is one of the most interesting cases of a quaternionic windowed Fourier transform since it is neither left- nor right-linear with respect to quaternionic constants (see [1], [2], [3]). This makes the study and, in particular, the construction of Gabor frames a challenge. Indeed, there does not exist yet a proper description of Gabor frames or links to Bargmann-Fock spaces ([4], [5]). In the past, studies related to Gabor frames where based on the real-valued inner product and used the rotational property of this inner product to make the Fourier transform one-sided. While several properties like uncertainty relations and the Balian-Low theorem could be shown in this case for a deeper discussion the application of the quaternionic inner product is necessary, see for instance [6], [7]. Yet, such an application does not allow to take advantage of the Hilbert space structure of the underlying function space [8]. Here, we are going a different way by using the duality of nonlinear modulation operators. 
This setting allows us to get properties like Wexler-Rax biorthogonality, RonShen duality, and Walnut and Janssen representations. This is a major step in working with quaternionic Gabor frames.

\section{Preliminaries}

Let $\mathbb{H}$ be the real quaternion algebra given by

$$
\mathbb{H}=\left\{q: q=q_{0}+q_{1} i+q_{2} j+q_{3} k, \quad q_{0}, q_{1}, q_{2}, q_{3} \in \mathbb{R}\right\},
$$

where the elements $i, j, k$ satisfy Hamiltons multiplication rules

$$
i j=-j i=k, \quad i^{2}=j^{2}=k^{2}=-1 .
$$

Every quaternion $q$ can be written as the sum of its scalar part $S c(q):=$ $q_{0} \in \mathbb{R}$ with its vectorial part $\operatorname{Vec}(q):=q_{1} i+q_{2} j+q_{3} k \in \mathbb{R}^{3}$ (or pure quaternion). The conjugation is an involutory automorphism $\cdot: \mathbb{H} \rightarrow \mathbb{H}$ defined as $q \mapsto \bar{q}=S c(q)-V e c(q)$. The modulus of a quaternion is defined by $|q|^{2}=q \bar{q}=\bar{q} q=q_{0}^{2}+q_{1}^{2}+q_{2}^{2}+q_{3}^{2}$.

We write a quaternion-valued function $f: \mathbb{R}^{2} \rightarrow \mathbb{H}$ as

$$
\mathbf{x}=\left(x_{1}, x_{2}\right) \mapsto f(\mathbf{x})=f_{0}(\mathbf{x})+f_{1}(\mathbf{x}) i+f_{2}(\mathbf{x}) j+f_{3}(\mathbf{x}) k,
$$

where $f_{0}, f_{1}, f_{2}, f_{3}: \mathbb{R}^{2} \rightarrow \mathbb{R}$ are its real-valued coefficient functions. We now introduce the necessary function spaces.

Definition 2.1 (Left module [9], [1]). We denote by $L^{p}\left(\mathbb{R}^{2}, \mathbb{H}\right), p \in \mathbb{N} \cup\{\infty\}$, the left module of all quaternion-valued functions $f: \mathbb{R}^{2} \rightarrow \mathbb{H}$ with finite $L^{p}-$ norm

$$
\|f\|_{p}=\left(\int_{\mathbb{R}^{2}}|f(\mathbf{x})|^{p} \mathrm{~d} \mathbf{x}\right)^{\frac{1}{p}}<\infty, \quad \text { if } p \in \mathbb{N},
$$

where $\mathrm{d} \mathbf{x}=\mathrm{d} x_{1} \mathrm{~d} x_{2}$ is the usual Lebesgue measure in $\mathbb{R}^{2}$, or

$$
\|f\|_{\infty}=\underset{\mathbf{x} \in \mathbb{R}^{2}}{\operatorname{ess} \sup }|f(\mathbf{x})|<\infty, \quad \text { if } p=\infty .
$$

Moreover, $L^{2}\left(\mathbb{R}^{2}, \mathbb{H}\right)$ is a Hilbert module with norm induced by the (quaternionic valued) inner product

$$
(f, g)_{2}:=\int_{\mathbb{R}^{2}} \overline{f(\mathbf{x})} g(\mathbf{x}) \mathrm{d} \mathbf{x} .
$$

In a similar way, we define the space $\ell^{p}(\mathbb{H}), p \in \mathbb{N}$, as the left module of all quaternion-valued sequences $\mathbf{c}=\left(c_{\mathbf{n}}\right)_{\mathbf{n} \in \mathbb{Z}^{2}}$ with finite $\ell^{p}$-norm

$$
\|\mathbf{c}\|_{\ell^{p}}=\left(\sum_{\mathbf{n} \in \mathbb{Z}^{2}}\left|c_{\mathbf{n}}\right|^{p}\right)^{\frac{1}{p}} .
$$

We conclude with the definition of the Wiener space. 
Definition 2.2 (Wiener space [10]). A function $g \in L^{\infty}\left(\mathbb{R}^{2}, \mathbb{R}\right)$ belongs to the Wiener space $W=W\left(\mathbb{R}^{2}\right)$ if

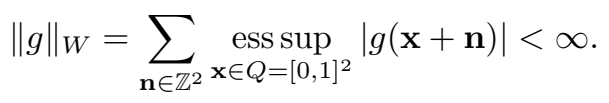

Functions in the Wiener space are locally bounded and in $L^{1}\left(\mathbb{R}^{2}, \mathbb{R}\right)$. The Wiener space is the choice space for window functions in Gabor analysis and it will be of importance in the next theorems and results.

\section{Windowed quaternionic Fourier transform (WQFT)}

We consider the windowed quaternionic Fourier transform (WQFT) based on the two sided quaternionic Fourier transform (QFT) which is discussed in $[1,2,3,6]$, among others. The problem with the two-sided WQFT is that it is neither left- nor right-linear with respect to quaternionic constants and, therefore, needs a special treatment. Hence, we introduce the following operators. For $\mathbf{b}=\left(b_{1}, b_{2}\right), \boldsymbol{\omega}=\left(\omega_{1}, \omega_{2}\right) \in \mathbb{R}^{2}$,

$$
\begin{gathered}
T_{\mathbf{b}} f(\mathbf{x})=f(\mathbf{x}-\mathbf{b}), \quad \text { translation by } \mathbf{b}, \\
E(f)=\int_{\mathbb{R}^{2}} f(\mathbf{x}) \mathrm{d} \mathbf{x}, \quad \text { mean operator, } \\
e_{\boldsymbol{\omega}}(f)(\mathbf{x})=e^{-2 \pi i x_{1} \omega_{1}} f(\mathbf{x}) e^{-2 \pi j x_{2} \omega_{2}}, \quad \text { modulation by } \boldsymbol{\omega} .
\end{gathered}
$$

In addition, we consider the modulation by $\mathbf{x}$ acting on $\mathbf{c}=\left(c_{\mathbf{k}}\right)_{\mathbf{k} \in \mathbb{Z}^{2}} \in \ell^{2}(\mathbb{H})$ as

$$
\mathbf{c} \mapsto e_{\mathbf{x}}^{\prime}(\mathbf{c})=\left(e^{-2 \pi i x_{1} k_{1}} c_{\mathbf{k}} e^{-2 \pi j x_{2} k_{2}}\right)_{\mathbf{k} \in \mathbb{Z}^{2}}, \quad \mathbf{k}=\left(k_{1}, k_{2}\right) .
$$

We remark that the operators $e_{\boldsymbol{\omega}}, e_{\mathbf{x}}^{\prime}$ are quaternionic non-linear operators.

We use these operators to describe the two-sided quaternionic Fourier transform (see, for example [1]).

Definition 3.1 (Two-sided quaternionic Fourier transform (QFT)). The two sided quaternionic Fourier transform of $f \in L^{1}\left(\mathbb{R}^{2}, \mathbb{H}\right)$ is defined as $\mathcal{F}_{q}(f)$ : $\mathbb{R}^{2} \rightarrow \mathbb{H}$, where

$$
\boldsymbol{\omega}=\left(\omega_{1}, \omega_{2}\right) \mapsto \mathcal{F}_{q}(f)(\boldsymbol{\omega})=\int_{\mathbb{R}^{2}} e^{-2 \pi i x_{1} \omega_{1}} f(\mathbf{x}) e^{-2 \pi j x_{2} \omega_{2}} \mathrm{~d} \mathbf{x}=E\left(e_{\boldsymbol{\omega}}(f)\right) .
$$

Furthermore, we reconstruct the signal $f$ by

$$
\begin{aligned}
\mathbf{x}=\left(x_{1}, x_{2}\right) \mapsto f(\mathbf{x})= & \mathcal{F}_{q}^{-1}\left(\mathcal{F}_{q} f\right)(\mathbf{x})=\int_{\mathbb{R}^{2}} e^{2 \pi i x_{1} \omega_{1}} \widehat{f}(\boldsymbol{\omega}) e^{2 \pi j x_{2} \omega_{2}} \mathrm{~d} \boldsymbol{\omega} \\
& =E\left(e_{-\boldsymbol{\omega}}\left(E\left(e_{\boldsymbol{\omega}}(f)\right)\right)\right) .
\end{aligned}
$$

Since the QFT only gives global information about the behaviour of $f$, we use a window function (or cut-off function) to obtain information on local properties of the signal [11]. 
Definition 3.2 (Windowed Quaternionic Fourier Transform). Let $g \in L^{2}\left(\mathbb{R}^{2}, \mathbb{R}\right)$ be a non-zero, real-valued window function. The windowed quaternionic Fourier transform (WQFT) of $f \in L^{2}\left(\mathbb{R}^{2}, \mathbb{H}\right)$ w.r.t. the window $g$ is defined as

$$
\begin{aligned}
\mathcal{Q}_{g} f(\mathbf{b}, \boldsymbol{\omega}) & =\int_{\mathbb{R}^{2}} e^{-2 \pi i x_{1} \omega_{1}} f(\mathbf{x}) g(\mathbf{x}-\mathbf{b}) e^{-2 \pi j x_{2} \omega_{2}} \mathrm{~d} \mathbf{x} \\
& =E\left(e_{\boldsymbol{\omega}}(f) T_{\mathbf{b}} g\right), \quad(\mathbf{b}, \boldsymbol{\omega}) \in \mathbb{R}^{2} \times \mathbb{R}^{2} .
\end{aligned}
$$

Given two non-zero, real-valued windows $g, \gamma \in L^{2}\left(\mathbb{R}^{2}, \mathbb{R}\right)$ which are not orthogonal to each other we have for the reconstruction of $f$ the expression

$$
\begin{aligned}
f(\mathbf{x}) & =\frac{1}{\left|(g, \gamma)_{2}\right|} \int_{\mathbb{R}^{2}} \int_{\mathbb{R}^{2}} e^{2 \pi i x_{1} \omega_{1}} \mathcal{Q}_{\gamma} f(\mathbf{b}, \boldsymbol{\omega}) g(\mathbf{x}-\mathbf{b}) e^{2 \pi j x_{2} \omega_{2}} \mathrm{~d} \boldsymbol{\omega} \mathrm{d} \mathbf{b} \\
& =\frac{1}{\left|(g, \gamma)_{2}\right|} \int_{\mathbb{R}^{2}} \int_{\mathbb{R}^{2}} e^{2 \pi i x_{1} \omega_{1}} \mathcal{Q}_{\gamma} f(\mathbf{b}, \boldsymbol{\omega}) e^{2 \pi j x_{2} \omega_{2}} g(\mathbf{x}-\mathbf{b}) \mathrm{d} \boldsymbol{\omega} \mathrm{d} \mathbf{b} \\
& =\frac{1}{\left|(g, \gamma)_{2}\right|} \int_{\mathbb{R}^{2}} \int_{\mathbb{R}^{2}} e_{-\boldsymbol{\omega}} \mathcal{Q}_{\gamma} f(\mathbf{b}, \boldsymbol{\omega}) T_{\mathbf{b}} g(\mathbf{x}) \mathrm{d} \boldsymbol{\omega} \mathrm{d} \mathbf{b} \\
& =\frac{1}{\left|(g, \gamma)_{2}\right|} \int_{\mathbb{R}^{2}} \int_{\mathbb{R}^{2}} e_{-\boldsymbol{\omega}} E\left(e_{\boldsymbol{\omega}}(f) T_{\mathbf{b}} g\right) T_{\mathbf{b}} g(\mathbf{x}) \mathrm{d} \boldsymbol{\omega} \mathrm{d} \mathbf{b} .
\end{aligned}
$$

\section{The structure of quaternionic Gabor frames}

In the previous section we presented the reconstruction of a signal from its continuous QWFT. However, a continuous reconstruction is not satisfactory for real-life purposes. Instead, a discrete reconstruction scheme is required based on a series expansion w.r.t. a countable subset of time-frequency shifts of the chosen windows (since $L^{2}(\mathbb{H}, \mathbb{R})$ is separable). This corresponds to replace the integrals by sums with coefficients on a sufficiently dense enough lattice which allows for the reconstruction of the original signal and leads to the idea of quaternionic Gabor frames. In what follows, let $g, \gamma \in L^{2}\left(\mathbb{R}^{2}, \mathbb{R}\right)$ be non-zero real-valued windows, and $\alpha, \beta>0$ be lattice parameters.

Definition 4.1 (Quaternionic frame operator). The quaternionic frame operator $S_{\gamma, g}$ is defined by

$$
S_{g, \gamma} f=\sum_{\mathbf{m} \in \mathbb{Z}^{2}} \sum_{\mathbf{n} \in \mathbb{Z}^{2}} e_{-\beta \mathbf{m}}^{\prime}\left(E\left(e_{\beta \mathbf{m}}\left(f T_{\alpha \mathbf{n}} \gamma\right)\right)\right) T_{\alpha \mathbf{n}} g .
$$

Traditionally the study of frames is done via investigation of the frame operator. Here, a problem arises since the frame operator is neither left- or right-linear (w.r.t. quaternions) which makes the direct application of Hilbert space methods impossible. To overcome this problem we introduce the concept of correlation function.

Definition 4.2 (Correlation function). The function

$$
G_{\mathbf{n}}(\mathbf{x})=\sum_{\mathbf{m} \in \mathbb{Z}^{2}} \gamma\left(\mathbf{x}-\frac{\mathbf{n}}{\beta}-\alpha \mathbf{m}\right) g(\mathbf{x}-\alpha \mathbf{m}),
$$


is called correlation function for $\mathbf{n} \in \mathbb{Z}^{2}$.

\subsection{Boundedness results for the quaternionic frame operator}

In the following lemma we show the boundedness of the correlation function $G_{\mathbf{n}}$ for the case of the windows $g$ and $\gamma$ in the Wiener space.

Lemma 4.3. If $g, \gamma \in W\left(\mathbb{R}^{2}\right)$, then $G_{\mathbf{n}} \in L^{\infty}\left(\mathbb{R}^{2}\right)$. Moreover, it holds

$$
\sum_{\mathbf{n} \in \mathbb{Z}^{2}}\left\|G_{\mathbf{n}}\right\|_{\infty} \leq\left(\frac{1}{\alpha}+1\right)^{2}(2 \beta+2)^{2}\|g\|_{W}\|\gamma\|_{W} .
$$

For the proof we refer to [10], Lemma 6.3.1.

Theorem 4.4 (Walnut theorem). The operator

$$
\begin{gathered}
S_{g, \gamma} f=\sum_{\mathbf{m}, \mathbf{n} \in \mathbb{Z}^{2}} e_{-\beta \mathbf{m}}^{\prime}\left(E\left(e_{\beta \mathbf{m}}\left(f T_{\alpha \mathbf{n}} \gamma\right)\right)\right) T_{\alpha \mathbf{n}} g \\
=\sum_{\mathbf{m}, \mathbf{n} \in \mathbb{Z}^{2}} e_{-\beta \mathbf{m}}^{\prime}\left(\int_{\mathbb{R}^{2}} e^{-2 \pi i x_{1} \beta m_{1}} f(\mathbf{x}) \gamma(\mathbf{x}-\alpha \mathbf{n}) e^{-2 \pi j x_{2} \beta m_{2}} d \mathbf{x}\right) T_{\alpha \mathbf{n}} g,
\end{gathered}
$$

can be written as

$$
S_{g, \gamma} f=\beta^{-2} \sum_{\mathbf{n} \in \mathbb{Z}^{2}} G_{\mathbf{n}} T_{\frac{\mathbf{n}}{\beta}} f .
$$

Proof. For every $f \in L^{2}\left(\mathbb{R}^{2}, \mathbb{H}\right)$ we have

$$
\int_{\mathbb{R}^{2}} e^{-2 \pi i \beta x_{1} m_{1}} f(\mathbf{x}) \gamma(\mathbf{x}-\alpha \mathbf{n}) e^{-2 \pi j \beta x_{2} m_{2}} \mathrm{~d} \mathbf{x} \in \ell^{2}(\mathbb{H}) .
$$

Therefore we can rewrite the elements

$$
\xi_{\mathbf{n}}=\sum_{\mathbf{m}} e_{-\beta \mathbf{m}}^{\prime} \int_{\mathbb{R}^{2}} e^{-2 \pi i \beta x_{1} m_{1}} f(\mathbf{x}) \gamma(\mathbf{x}-\alpha \mathbf{n}) e^{-2 \pi j \beta x_{2} m_{2}} \mathrm{~d} \mathbf{x},
$$

with the Poisson summation formula for the two sided QFT (see [7]) as

$$
\xi_{\mathbf{n}}=\beta^{-2} \sum_{\mathbf{m}}\left(f T_{\alpha \mathbf{n}} \gamma\right)\left(\mathbf{x}-\frac{\mathbf{m}}{\beta}\right) .
$$

Hence, the quaternionic frame operator becames

$$
S_{g, \gamma} f=\sum_{\mathbf{m}}\left(\sum_{\mathbf{n}} \beta^{-2} f\left(\mathbf{x}-\frac{\mathbf{n}}{\beta}\right) \gamma\left(\mathbf{x}-\alpha \mathbf{m}-\frac{\mathbf{n}}{\beta}\right)\right) g(\mathbf{x}-\alpha \mathbf{m}) .
$$

We assume $f$ to have compact support. Then, by interchanging summation we obtain

$$
\begin{gathered}
S_{g, \gamma} f=\beta^{-2} \sum_{\mathbf{n}}\left(\sum_{\mathbf{m}} \gamma\left(\mathbf{x}-\alpha \mathbf{m}-\frac{\mathbf{n}}{\beta}\right) g(\mathbf{x}-\alpha \mathbf{m})\right) f\left(\mathbf{x}-\frac{\mathbf{n}}{\beta}\right) \\
=\beta^{-2} \sum_{\mathbf{n}} G_{\mathbf{n}} T_{\mathbf{n} / \beta} f,
\end{gathered}
$$

so the result holds for functions with compact support. The extension to $L^{2}$ follows by density arguments, and this since both $g$ and $\gamma$ are real-valued. 
Based on Lemma 4.3 we proof the boundedness of the quaternionic frame operator $S_{g, \gamma}$. Indeed,

$$
\begin{gathered}
\left\|S_{g, \gamma} f\right\|_{p} \leq \beta^{-2} \sum_{\mathbf{n}}\left\|G_{\mathbf{n}} T_{\mathbf{n} / \beta} f\right\|_{p} \leq \beta^{-2} \sum_{\mathbf{n}}\left\|G_{\mathbf{n}}\right\|_{\infty}\left\|T_{\mathbf{n} / \beta} f\right\|_{p} \\
=\beta^{-2} \sum_{\mathbf{n}}\left\|G_{\mathbf{n}}\right\|_{\infty}\|f\|_{p} \leq \beta^{-2}\left(\frac{1}{\alpha}+1\right)^{2}(2 \beta+2)^{2}\|g\|_{W}\|\gamma\|_{W}\|f\|_{p} .
\end{gathered}
$$

Theorem 4.5. For all $f, h \in L^{2}(\mathbb{R}, \mathbb{H})$, we have

$$
\left(S_{g, \gamma} f, h\right)_{2}=\beta^{-2} \sum_{\mathbf{m}, \mathbf{l} \in \mathbb{Z}^{2}} \int_{Q_{1 / \beta}} G_{\mathbf{m}, \mathbf{l}}(\mathbf{x}) T_{\mathbf{l} / \beta} f(\mathbf{x}) \overline{T_{\mathbf{m} / \beta} h(\mathbf{x})} d \mathbf{x}
$$

where $Q_{1 / \beta}$ is a two-dimensional cube with side-length $1 / \beta$ and $G_{\mathbf{m}, 1}$ is given as

$$
G_{\mathbf{m}, \mathbf{l}}(\mathbf{x}):=\sum_{\mathbf{k} \in \mathbb{Z}^{2}} \gamma\left(\mathbf{x}-\frac{\mathbf{m}}{\beta}-\alpha \mathbf{k}\right) g\left(\mathbf{x}-\frac{\mathbf{l}}{\beta}-\alpha \mathbf{k}\right) .
$$

Proof. First, we assume $f$ and $h$ to be bounded and to have compact support. Hence, the functions $T_{\mathbf{m} / \beta} f$ and $T_{\mathbf{m} / \beta} h, \mathbf{m} \in \mathbb{Z}^{2}$, have compact support. By Theorem 4.4 for $S_{g, \gamma} f$ we obtain

$$
\begin{gathered}
\left(S_{g, \gamma} f, h\right)_{2}=\beta^{-2} \int_{\mathbb{R}^{2}}\left(\sum_{\mathbf{n} \in \mathbb{Z}^{2}} G_{\mathbf{n}}(\mathbf{x}) f\left(\mathbf{x}-\frac{\mathbf{n}}{\beta}\right)\right) \overline{h(\mathbf{x})} \mathrm{d} \mathbf{x} \\
=\beta^{-2} \int_{Q_{1 / \beta}} \sum_{\mathbf{m} \in \mathbb{Z}^{2}} \sum_{\mathbf{n} \in \mathbb{Z}^{2}} G_{\mathbf{n}}\left(\mathbf{x}-\frac{\mathbf{m}}{\beta}\right) f\left(\mathbf{x}-\frac{\mathbf{m}+\mathbf{n}}{\beta}\right) \overline{h\left(\mathbf{x}-\frac{\mathbf{m}}{\beta}\right)} \mathrm{d} \mathbf{x} .
\end{gathered}
$$

Due to the compact support of the functions, the sums over $\mathbf{m}$ and $\mathbf{n}$ are finite. The change of variable $\mathbf{l}=\mathbf{m}+\mathbf{n}$ and the fact that $G_{\mathbf{n}}\left(\mathbf{x}-\frac{\mathbf{j}}{\beta}\right)=G_{\mathbf{j}, \mathbf{j}+\mathbf{n}}(\mathbf{x})$ gives

$$
\left(S_{g, \gamma} f, h\right)_{2}=\beta^{-2} \int_{Q} \sum_{\mathbf{m}, \mathbf{l} \in \mathbb{Z}^{2}} G_{\mathbf{m}, \mathbf{l}}(\mathbf{x}) T_{\mathbf{l} / \beta} f(\mathbf{x}) \overline{T_{\mathbf{m} / \beta} h(\mathbf{x})} \mathrm{d} \mathbf{x} .
$$

For the extension to $L^{2}$, we introduce the operator $G(\mathbf{x}), \mathbf{x} \in \mathbb{R}^{2}$, acting on finite sequences $\mathbf{c}=\left(c_{\mathbf{l}}\right)_{\mathbf{l} \in \mathbb{Z}^{2}}$ by the matrix multiplication

$$
(G(\mathbf{x}) \mathbf{c})_{\mathbf{m}}=\sum_{\mathbf{l}} G_{\mathbf{m}, \mathbf{l}}(\mathbf{x}) c_{\mathbf{l}}
$$

If $g, \gamma \in W\left(\mathbb{R}^{2}\right)$ by Schur's Test ([10], Lemma 6.2.1) we get

$$
\begin{gathered}
\sum_{\mathbf{l}}\left|G_{\mathbf{m}, \mathbf{l}}(\mathbf{x})\right| \leq \sum_{\mathbf{l}} \sum_{\mathbf{k}}\left|g\left(\mathbf{x}-\frac{\mathbf{l}}{\beta}-\alpha \mathbf{k}\right)\right| \mid \gamma\left(\mathbf{x}-\frac{\mathbf{m}}{\beta}-\alpha \mathbf{k} \mid\right. \\
=\sum_{\mathbf{k}}\left(\sum_{\mathbf{l}}\left|g\left(\mathbf{x}-\frac{\mathbf{l}}{\beta}-\alpha \mathbf{k}\right)\right|\right)\left|\gamma\left(\mathbf{x}-\frac{\mathbf{m}}{\beta}-\alpha \mathbf{k}\right)\right| \\
\leq(\beta+1)^{2}\left(\frac{1}{\alpha}+1\right)^{2}\|g\|_{W}\|\gamma\|_{W} .
\end{gathered}
$$


Of course, a similar estimate holds also for $\sum_{\mathbf{m}}\left|G_{\mathbf{m}, \mathbf{l}}(\mathbf{x})\right|$. Hence, $G(\mathbf{x})$ defines a bounded operator on $\ell^{p}, 1 \leq p \leq \infty$. If $f, h \in L^{2}$, then both sequences $\left\{f\left(\mathbf{x}-\frac{\mathbf{l}}{\beta}\right), \mathbf{l} \in \mathbb{Z}^{2}\right\}$ and $\left\{h\left(\mathbf{x}-\frac{\mathbf{l}}{\beta}\right), \mathbf{l} \in \mathbb{Z}^{2}\right\}$ are in $\ell^{2}(\mathbb{H})$ for almost all $\mathbf{x} \in \mathbb{R}^{2}$, and the matrix representation holds.

At this point a few remarks are necessary. First, for $g, \gamma \in L^{2}\left(\mathbb{R}^{2}\right)$ we have $T_{\frac{\mathbf{m}}{\beta}} \gamma, T_{\frac{1}{\beta}} g \in L^{1}\left(\mathbb{R}^{2}\right)$ so that $G_{\mathbf{m}, \mathbf{l}}(\mathbf{x}) \in L^{1}\left(Q_{\alpha}\right)$ for all $\mathbf{m}, \mathbf{l} \in \mathbb{Z}^{2}$. Second, if $g=\gamma$ then $G(\mathbf{x})=\left(G_{\mathbf{m}, \mathbf{l}}(\mathbf{x})\right)_{\mathbf{m}, \mathbf{l}}$ defines a positive operator, that is,

$$
\begin{gathered}
(G(\mathbf{x}) \mathbf{c}, \mathbf{c})_{\ell^{2}}=\sum_{\mathbf{m}, \mathbf{l} \in \mathbb{Z}^{2}} \sum_{\mathbf{k} \in \mathbb{Z}^{2}} \overline{c_{\mathbf{m}}} \gamma\left(\mathbf{x}-\frac{\mathbf{m}}{\beta}-\alpha \mathbf{k}\right) \gamma\left(\mathbf{x}-\frac{\mathbf{l}}{\beta}-\alpha \mathbf{k}\right) c_{\mathbf{l}} \\
=\sum_{\mathbf{k} \in \mathbb{Z}^{2}} \overline{\left(\sum_{\mathbf{m} \in \mathbb{Z}^{2}} \gamma\left(\mathbf{x}-\frac{\mathbf{m}}{\beta}-\alpha \mathbf{k}\right) c_{\mathbf{m}}\right)}\left(\sum_{\mathbf{l} \in \mathbb{Z}^{2}} \gamma\left(\mathbf{x}-\frac{\mathbf{l}}{\beta}-\alpha \mathbf{k}\right) c_{\mathbf{l}}\right) \\
=\sum_{\mathbf{k} \in \mathbb{Z}^{2}}\left|\sum_{\mathbf{l} \in \mathbb{Z}^{2}} \gamma\left(\mathbf{x}-\frac{\mathbf{l}}{\beta}-\alpha \mathbf{k}\right) c_{\mathbf{l}}\right|^{2} \geq 0 .
\end{gathered}
$$

In the next lemmas we prove some results regarding the boundedness and invertibility of the quaternionic Gabor frame.

Theorem 4.6. Let $I$ denote the identity operator in $\ell^{2}(\mathbb{H})$.

1. $S_{\gamma, \gamma}$ is invertible on $L^{2}\left(\mathbb{R}^{2}, \mathbb{H}\right)$, if and only if there exists a constant $a>0$ such that $G(\mathbf{x}) \geq a I$ for almost all $\mathbf{x} \in \mathbb{R}^{2}$.

2. $S_{\gamma, \gamma}$ is a bounded operator on $L^{2}\left(\mathbb{R}^{2}, \mathbb{H}\right)$ if and only if there exists a constant $b>0$ such that $G(\mathbf{x}) \leq b I$ for almost all $\mathbf{x} \in \mathbb{R}^{2}$.

Proof. For the first statement, we assume that $G(\mathbf{x}) \geq a I$. Then we have for every $f \in L^{\infty}\left(\mathbb{R}^{2}, \mathbb{H}\right)$ with compact support that

$$
\left|\sum_{\mathbf{m}, \mathbf{n}} G_{\mathbf{m}, \mathbf{n}}\left(T_{\mathbf{m}} f\right) \overline{\left(T_{\mathbf{n}} f\right)}\right| \geq a \sum_{\mathbf{n}}\left|T_{\mathbf{n}} f\right|^{2} .
$$

We integrate over $Q_{1 / \beta}$ and use Theorem 4.5 and we obtain

$$
\left(S_{\gamma, \gamma} f, f\right)_{2} \geq a \beta^{-2} \int_{Q_{1 / \beta}} \sum_{\mathbf{n}}\left|T_{\mathbf{n}} f\right|^{2}=a \beta^{-2}\|f\|_{2}^{2} .
$$

By density arguments the result holds for all $f \in L^{2}\left(\mathbb{R}^{2}, \mathbb{H}\right)$ and, therefore, $S_{\gamma, \gamma}$ is invertible there.

Conversely, if $S_{\gamma, \gamma}$ is not invertible then, there exists a sequence of bounded functions with compact support in $L^{2}\left(\mathbb{R}^{2}, \mathbb{H}\right)$ such that

$$
\left(S_{\gamma, \gamma} f_{k}, f_{k}\right)_{2}<\frac{1}{k}\left\|f_{k}\right\|_{2}^{2}
$$

By Theorem 4.5 this implies

$$
\int_{Q_{1 / \beta}}\left(\frac{1}{k} \sum_{\mathbf{m}}\left|f_{k}(\mathbf{x}-\mathbf{m})\right|^{2}-\beta^{-2} \sum_{\mathbf{m}, \mathbf{n}} G_{\mathbf{m}, \mathbf{n}} f_{k}(\mathbf{x}-\mathbf{m}) \overline{f_{k}(\mathbf{x}-\mathbf{n})}\right) \mathrm{d} \mathbf{x}>0 .
$$


Therefore, there exists a sequence of sets $E_{k} \subseteq Q_{1 / \beta}$ with positive measure and such that for $\mathbf{x} \in E_{k}$ we have

$$
\frac{1}{k} \sum_{\mathbf{m}}\left|f_{k}(\mathbf{x}-\mathbf{m})\right|^{2}-\sum_{\mathbf{m}, \mathbf{n}} G_{\mathbf{m}, \mathbf{n}} f_{k}(\mathbf{x}-\mathbf{m}) \overline{f_{k}(\mathbf{x}-\mathbf{n})}>0 .
$$

Hence, we get

$$
\inf _{\|\mathbf{c}\|_{\ell^{2}}^{2} \leq 1}(G(\mathbf{x}) \mathbf{c}, \mathbf{c})_{\ell^{2}}<\frac{\beta^{2}}{k},
$$

for $\mathbf{x} \in E_{k}$, and a uniform inequality $a I<G(\mathbf{x})$ cannot hold for a.a. $\mathbf{x}$.

The proof of statement 2 . is similar and will be omitted.

Lemma 4.7. If $S_{g, \gamma}$ is bounded on $L^{2}\left(\mathbb{R}^{2}, \mathbb{H}\right)$, then we have

$$
\left(S_{g, \gamma} f, h\right)_{2}=\beta^{-2}\left(\sum_{\mathbf{n}} G_{\mathbf{n}} T_{\mathbf{n} / \beta} f, h\right)_{2},
$$

for all $f, h \in L^{\infty}\left(\mathbb{R}^{2}, \mathbb{H}\right)$ with compact support.

The proof follows the same lines as the one of Theorem 4.5 under slightly different assumptions.

Lemma 4.8. If $S_{g, \gamma}$ is bounded then for all $\mathbf{n} \in \mathbb{Z}^{2}$ it holds

$$
\left\|G_{\mathbf{n}}\right\|_{\infty} \leq \beta^{2}\left\|S_{g, \gamma}\right\|_{o p} .
$$

Proof. Choose $f, h \in L^{\infty}\left(\mathbb{R}^{2}, \mathbb{H}\right)$, with support in $Q_{1 / \beta}$ and let $\mathbf{l}, \mathbf{m} \in \mathbb{Z}^{2}$ be arbitrary. Due to the boundedness of $S_{g, \gamma}$, we have

$$
\left|\left(S_{g, \gamma} T_{\mathbf{l} / \beta} f, T_{\mathbf{m} / \beta} h\right)_{2}\right| \leq\left\|S_{g, \gamma}\right\|_{o p}\|f\|_{2}\|h\|_{2} .
$$

Due to Lemma 4.7 we get

$$
\begin{gathered}
\left(S_{g, \gamma} T_{\mathbf{l} / \beta} f, T_{\mathbf{m} / \beta} h\right)_{2}=\beta^{-2}\left(\sum_{\mathbf{n} \in \mathbb{Z}^{2}} G_{\mathbf{n}} T_{\frac{\mathbf{n}}{\beta}}\left(T_{\frac{1}{\beta}} f\right), T_{\frac{\mathbf{m}}{\beta}} h\right)_{2} \\
=\beta^{-2}\left(\sum_{\mathbf{n} \in \mathbb{Z}^{2}} G_{\mathbf{n}} T_{\frac{\mathbf{n}+1}{\beta}} f, T_{\frac{\mathbf{m}}{\beta}} h\right)_{2} .
\end{gathered}
$$

Since the supports of $T_{\frac{\mathbf{n}+1}{\beta}} f$ and $T_{\frac{\mathbf{m}}{\beta}} h$ are pairwise disjoint in general, only the term $\mathbf{n}+\mathbf{l}=\mathbf{m}$ survives. Combining (4.4) and (4.5), we obtain

$$
\beta^{-2}\left|\int_{Q_{1 / \beta}} G_{\mathbf{m}-\mathbf{l}}\left(\mathbf{x}+\frac{\mathbf{m}}{\beta}\right) f(\mathbf{x}) \overline{h(\mathbf{x})} \mathrm{d} \mathbf{x}\right| \leq\left\|S_{g, \gamma}\right\|_{o p}\|f\|_{2}\|h\|_{2},
$$

for all $f, h \in L^{\infty}\left(\mathbb{R}^{2}, \mathbb{H}\right) \subseteq L^{2}\left(\mathbb{R}^{2}, \mathbb{H}\right)$ and all $\mathbf{l}, \mathbf{m} \in \mathbb{Z}^{2}$. The argument of density extends $(4.6)$ to $f, h \in L^{2}\left(Q_{1 / \beta}, \mathbb{H}\right)$, and we have

$$
\beta^{-2} \operatorname{ess} \sup _{\mathbf{x} \in Q_{1 / \beta}}\left|G_{\mathbf{n}}\left(\mathbf{x}+\frac{\mathbf{n}+\mathbf{1}}{\beta}\right)\right| \leq\left\|S_{g, \gamma}\right\|_{o p},
$$

for all $\mathbf{l} \in \mathbb{Z}^{2}$. Therefore,

$$
\left\|G_{\mathbf{n}}\right\|_{\infty} \leq \beta^{2}\left\|S_{g, \gamma}\right\|_{o p} .
$$




\subsection{Janssen's representation}

In order to obtain the Janssen's representation for the correlation function $G_{\mathbf{n}}$ we first compute its l-th discrete QFT coefficients

$$
\begin{gathered}
\widehat{G_{\mathbf{n}}(\mathbf{l})}=\alpha^{-2} \int_{Q_{\alpha}} e^{-2 \pi i l_{1} x_{1} / \alpha} G_{\mathbf{n}}(\mathbf{x}) e^{-2 \pi j l_{2} x_{2} / \alpha} \mathrm{d} \mathbf{x} \\
=\alpha^{-2} \int_{Q_{\alpha}} \sum_{\mathbf{m} \in \mathbb{Z}^{2}} e^{-2 \pi i l_{1} x_{1} / \alpha}\left(\left(T_{\mathbf{n} / \beta} g\right) \gamma\right)(\mathbf{x}-\alpha \mathbf{m}) e^{-2 \pi j l_{2} x_{2} / \alpha} \mathrm{d} \mathbf{x} \\
=\alpha^{-2} \int_{\mathbb{R}^{2}} e^{-2 \pi i l_{1} x_{1} / \alpha}\left(\left(T_{\mathbf{n} / \beta} g\right) \gamma\right)(\mathbf{x}) e^{-2 \pi j l_{2} x_{2} / \alpha} \mathrm{d} \mathbf{x} \\
=\alpha^{-2} E\left(e_{\mathbf{l} / \alpha}\left(\left(T_{\mathbf{n} / \beta} g\right) \gamma\right)\right),
\end{gathered}
$$

where $\left(T_{\mathbf{n} / \beta} g\right) \gamma$ denotes the pointwise multiplication of both functions. By Lemma $4.8 G_{\mathbf{n}}(\mathbf{x})$ has the following Fourier expansion

$$
G_{\mathbf{n}}(\mathbf{x})=\alpha^{-2} \sum_{\mathbf{l} \in \mathbb{Z}^{2}} e^{2 \pi i l_{1} x_{1} / \alpha} E\left(e_{l / \alpha}\left(\left(T_{\mathbf{n} / \beta} g\right) \gamma\right)\right) e^{2 \pi j l_{2} x_{2} / \alpha} .
$$

At this point, we should remark that it is not clear that (4.8) converges for general $g, \gamma \in L^{2}$. Thus, we employ the definition of TolimIeri and Orr.

Definition 4.9 (condition $\left.\left(\mathbf{A}^{\prime}\right)\right)$. A pair $(g, \gamma)$ of real-valued functions in $L^{2}\left(\mathbb{R}^{2}\right)$ satisfies condition $\left(\mathrm{A}^{\prime}\right)$ for the parameters $\alpha, \beta>0$ if

$$
\sum_{\mathbf{l}, \mathbf{n} \in \mathbb{Z}^{2}}\left|E\left(e_{\mathbf{l} / \alpha}\left(\left(T_{\mathbf{n} / \beta} g\right) \gamma\right)\right)\right|<\infty
$$

Replacing now $G_{\mathbf{n}}(\mathbf{x})$ in Theorem 4.4 (Walnut's Theorem) leads to the following theorem.

Theorem 4.10 (Janssen's Representation). Suppose that $(g, \gamma)$ satisfies condition ( $\left.A^{\prime}\right)$ for a given choice of $\alpha, \beta>0$. Then

$$
S_{g, \gamma}=(\alpha \beta)^{-2} \sum_{\mathbf{l}, \mathbf{n} \in \mathbb{Z}^{2}} e^{2 \pi i l_{1} x_{1} / \alpha} E\left(e_{\mathbf{l} / \alpha}\left(\left(T_{\mathbf{n} / \beta} g\right) \gamma\right)\right) e^{2 \pi j l_{2} x_{2} / \alpha} T_{\mathbf{n} / \beta},
$$

with absolute convergence in operator norm.

\subsection{Wexler-Raz Biorthogonality}

Theorem 4.11 (Wexler-Raz biorthogonality). Let both $S_{g, \gamma}$ and $S_{\gamma, g}$ be bounded operators on $L^{2}\left(\mathbb{R}^{2}, \mathbb{H}\right)$. Then the following conditions are equivalent:

1. $S_{g, \gamma}=S_{\gamma, g}=I d$ on $L^{2}\left(\mathbb{R}^{2}, \mathbb{H}\right)$;

2. $(\alpha \beta)^{-2} E\left(e_{\mathbf{l} / \alpha}\left(\left(T_{\mathbf{n} / \beta} g\right) \gamma\right)\right)=\delta_{\mathbf{l}, 0} \delta_{\mathbf{n}, 0}$, for all $\mathbf{l}, \mathbf{n} \in \mathbb{Z}^{2}$.

Proof. 1. $\Rightarrow$ 2. If $S_{g, \gamma}=S_{\gamma, g}=I d$, then, for arbitrary $f, h \in L^{\infty}\left(Q_{1 / \beta}\right)$ and $\mathbf{l}, \mathbf{m} \in \mathbb{Z}^{2}$ we get

$$
\begin{gathered}
\delta_{\mathbf{l}, \mathbf{m}}(f, h)_{2}=\left(S_{g, \gamma} T_{\mathbf{l} / \beta} f, T_{\mathbf{m} / \beta} h\right)_{2}=\beta^{-2}\left(\sum_{\mathbf{n} \in \mathbb{Z}^{2}} G_{\mathbf{n}} T_{\frac{\mathbf{n}+\mathbf{1}}{\beta}} f, T_{\mathbf{m} / \beta} h\right)_{2} \\
=\beta^{-2}\left(G_{\mathbf{m}-\mathbf{l}} T_{\mathbf{m} / \beta} f, T_{\mathbf{m} / \beta} h\right)_{2}=\beta^{-2}\left(\left(T_{-\mathbf{m} / \beta} G_{\mathbf{m}-\mathbf{l}}\right) f, h\right)_{2} .
\end{gathered}
$$


By usual density arguments this equality extends to $f, h \in L^{2}\left(Q_{1 / \beta}\right)$, and so it holds $\beta^{-2} G_{\mathbf{m}-\mathbf{l}}\left(\mathbf{x}+\frac{\mathbf{m}}{\beta}\right)=\delta_{\mathbf{l}, \mathbf{m}}$ for almost all $\mathbf{x} \in Q_{1 / \beta}$. In particular, we have $\beta^{-2} G_{0}(\mathbf{x})=1$ and $G_{\mathbf{n}}(\mathbf{x})=0$, if $\mathbf{n} \neq 0$ for a.a. $\mathbf{x} \in \mathbb{R}^{2}$. Since $G_{\mathbf{n}} \in L^{\infty}\left(Q_{\alpha}\right)$ has the Fourier series (4.8)

$$
G_{\mathbf{n}}(x)=\alpha^{-2} \sum_{\mathbf{l} \in \mathbb{Z}^{2}} e^{2 \pi i l_{1} x_{1} / \alpha} E\left(e_{\mathbf{l} / \alpha}\left(T_{\mathbf{n} / \beta} g \cdot \gamma\right)\right) e^{2 \pi j l_{2} x_{2} / \alpha},
$$

we conclude, by the uniqueness of Fourier coefficients, that

$$
(\alpha \beta)^{-2} E\left(e_{\mathbf{l} / \alpha}\left(T_{\mathbf{n} / \beta} g \cdot \gamma\right)\right)=\delta_{\mathbf{l}, 0} \delta_{\mathbf{n}, 0} .
$$

2 . $\Rightarrow 1$. If 2 . is satisfied then, the pair of window functions $(g, \gamma)$ fulfils condition (A') and, therefore, the representation (4.10) of $S_{g, \gamma}$ converges in the operator norm, with $S_{g, \gamma}=I d$.

Theorem 4.12. A quaternionic Gabor system

$$
\mathcal{G}(g, \alpha, \beta)=\left\{e_{\alpha \mathbf{m}}^{\prime}(\cdot) T_{\beta \mathbf{n}} g, \mathbf{m}, \mathbf{n} \in \mathbb{Z}^{2}\right\},
$$

of operators acting on $\ell^{2}(\mathbb{H})$ is a tight frame if and only if $\mathcal{G}(g, 1 / \beta, 1 / \alpha)$ is an orthonormal system. Moreover, the frame bound $A$ satisfies $A=(\alpha \beta)^{-2}\|g\|_{2}^{2}$.

Proof. The frame operator of a tight frame is a multiple identity. In particular, we have $\frac{1}{A} S_{g, g}=I d$. Using $\gamma=\frac{1}{A} g$ in Theorem 4.11, condition 2. we obtain

$$
(\alpha \beta)^{-2} \frac{1}{A} \int_{\mathbb{R}^{2}} e^{-2 \pi l_{1} x_{1} / \alpha}\left(T_{\mathbf{n} / \beta} g(\mathbf{x}) \cdot g(\mathbf{x})\right) e^{-2 \pi j l_{2} x_{2} / \alpha} \mathrm{d} \mathbf{x}=\delta_{\mathbf{l}, 0} \delta_{\mathbf{n}, 0} .
$$

In particular, for $\mathbf{l}=\mathbf{n}=0$, we get

$$
(\alpha \beta)^{-2} \frac{1}{A} \int_{\mathbb{R}^{2}}|g(\mathbf{x})|^{2} \mathrm{~d} \mathbf{x}=1 .
$$

Hence, $A=(\alpha \beta)^{-2}\|g\|_{2}^{2}$ for the frame bound.

Conversely, if $\mathcal{G}(g, 1 / \beta, 1 / \alpha)$ is an orthogonal system, then we obtain $S_{g, g}=$ $(\alpha \beta)^{-2}\|g\|_{2}^{2} I d$ by Janssen's representation. Therefore, $\mathcal{G}(g, \alpha, \beta)$ is a tight frame.

\section{Acknowledgment}

The work of the first author was supported by Portuguese funds through the CIDMA - Center for Research and Development in Mathematics and Applications, and the Portuguese Foundation for Science and Technology ("FCT-Fundação para a Ciência e a Tecnologia"), within project UID/MAT/ $0416 / 2013$.

\section{References}

[1] E. Hitzer, Quaternion Fourier Transform on Quaternion Fields and Generalizations, Advances in Applied Clifford Algebras, 17 (2007), 497-517. 
[2] M. Bahri, R. Ashino, Two-Dimensional Quaternionic Windowed Fourier Transform, Fourier Transforms - Approach to Scientific Principles, Prof. Goran Nikolic (Ed.), (2011), 247-260.

[3] M. Bahri, E. Hitzer, R. Ashino, R. Vaillancourt, Windowed Fourier transform of two-dimensional quaternionic signals, Applied Mathematics and Computation, 216 (2010), 2366-2379.

[4] L. D. Abreu, Sampling and interpolation in Bargmann-Fock spaces of polyanalytic functions, Applied and Computational Harmonic Analysis, 29 (2010), 287302.

[5] L. D. Abreu, K. Gröchenig, Banach Gabor frames with Hermite functions: polyanalytic spaces from the Heisenberg group, Applicable Analysis, 91 (2012), 19811997.

[6] Y. Fu, U. Kähler, P. Cerejeiras, The Balian-Low Theorem for the Windowed Quaternionic Fourier Transform, Advances in Applied Clifford Algebras, 22 (2012), 1025-1040.

[7] S. Hartmann, Quaternionic Gabor Expansion, Technische Universität Bergakademie Freiberg, 2015.

[8] T. A. Ell, N. LeBihan, S. J. Sangwine, Quaternion Fourier Transforms for Signal and Image Processing, John Wiley and Sons, New York, 2014.

[9] R. Delanghe, F. Sommen, V. Souček, Clifford algebra and spinor-valued functions: a function theory for the Dirac operator, Springer Science + Business Media, BV, 1992.

[10] K. Gröchenig, Foundations of Time-Frequency Analysis, Springer Science + Business Media, Berlin Heidelberg, 2001.

[11] S. Hartmann, Some Results on the Lattice Parameters of Quaternionic Gabor Frames, Advances in Applied Clifford Algebras, 26 (2016), 137-149.

Paula Cerejeiras

CIDMA - Center for Research and Development in Mathematics and Applications, Department of Mathematics, University of Aveiro

Campus Universitário de Santiago

3810-193 Aveiro, Portugal

e-mail: pceres@ua.pt

Stefan Hartmann

CIDMA - Center for Research and Development in Mathematics and Applications, Department of Mathematics, University of Aveiro (former student)

Campus Universitário de Santiago

3810-193 Aveiro, Portugal

e-mail: Hartmann. Aveiro@web.de

Heikki Orelma

Laboratory of Civil Engineering,

Tampere University of Technology,

Korkeakoulunkatu 10,

33720 Tampere, Finland

e-mail: heikki.orelma@tut.fi 